Western University

Scholarship@Western

$5-20-2019$

Effect of Learning to Use a Mobility Aid on Gait and Cognitive Demands in People with Mild to Moderate Alzheimer's Disease:

Part I - Cane

Susan W. Hunter

Alison Divine

Humberto Omana

Walter Wittich

Keith D Hill

See next page for additional authors

Follow this and additional works at: https://ir.lib.uwo.ca/ptpub

Part of the Physical Therapy Commons 


\section{Authors}

Susan W. Hunter, Alison Divine, Humberto Omana, Walter Wittich, Keith D Hill, Andrew M Johnson, and Jeffrey D Holmes 


\title{
Effect of Learning to Use a Mobility Aid on Gait and Cognitive Demands in People with Mild to Moderate Alzheimer's Disease: Part I - Cane
}

\author{
Susan W. Hunter ${ }^{\mathrm{a}, *}$, Alison Divine ${ }^{\mathrm{b}}$, Humberto Omana ${ }^{\mathrm{c}}$, Walter Wittich ${ }^{\mathrm{d}}$, Keith D. Hille ${ }^{\mathrm{e}}$, \\ Andrew M. Johnson ${ }^{\mathrm{f}}$ and Jeffrey D. Holmes ${ }^{\mathrm{g}}$

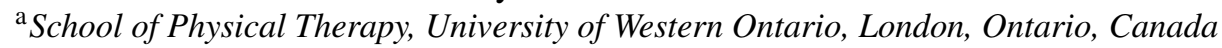 \\ ${ }^{\mathrm{b}}$ Faculty of Sport and Exercise Psychology, University of Leeds, Leeds, England \\ ${ }^{\mathrm{c}}$ Faculty of Health Sciences, University of Western Ontario, London, Ontario, Canada \\ ${ }^{\mathrm{d}}$ École d'optométrie, Université de Montréal, Montreal, Quebec, Canada \\ ${ }^{\mathrm{e}}$ School of Physiotherapy and Exercise Science, Curtin University, Perth, Australia \\ ${ }^{\mathrm{f}}$ School of Health Studies, University of Western Ontario, London, Ontario, Canada \\ ${ }^{\mathrm{g}}$ School of Occupational Therapy, University of Western Ontario, London, Ontario, Canada
}

Accepted 29 March 2019

\begin{abstract}
.
Background: People with Alzheimer's disease (AD) exhibit balance and walking impairments that increase falls risk. Prescription of a mobility aid is done to improve stability, yet also requires increased cognitive resources. Single-point canes require unique motor sequencing for safe use. The effect of learning to use a single-point cane has not been evaluated in people with AD.

Objectives: In people with $\mathrm{AD}$ and healthy adult controls: 1) examine changes in gait while using a cane under various walking conditions; and 2) determine the cognitive and gait costs associated with concurrent cane walking while multi-tasking.

Methods: Seventeen participants with AD (age $82.1 \pm 5.6$ years) and 25 healthy controls (age $70.8 \pm 14.1$ years) walked using a single-point cane in a straight (6 meter) and a complex (Figure of 8) path under three conditions: single-task (no aid), dual-task (walking with aid), and multi-task (walking with aid while counting backwards by ones). Velocity and stride time variability were recorded with accelerometers.

Results: Gait velocity significantly slowed for both groups in all conditions and stride time variability was greater in the AD group. Overall, multi-tasking produced a decrease in gait and cognitive demands for both groups, with more people with AD self-prioritizing the cognitive task over the gait task.

Conclusion: Learning to use a cane demands cognitive resources that lead to detrimental changes in velocity and stride time variability. This was most pronounced in people with mild to moderate AD. Future research needs to investigate the effects of mobility aid training on gait performance.
\end{abstract}

Keywords: Aged, assistive devices, cane, dementia, gait

\section{INTRODUCTION}

In addition to cognitive decline, Alzheimer's disease (AD) adversely affects balance and walking, two

${ }^{*}$ Correspondence to: Dr. Susan W. Hunter, University of Western Ontario, School of Physical Therapy, Room 1588, Elborn College, London, ON N6G 1H1, Canada. Tel.: +1 519661 2111/Ext. 82754; E-mail: susan.hunter@uwo.ca. prominent factors associated with an increased risk of falls [1]. The incidence of falls among those with $\mathrm{AD}$ is almost twice that of healthy older adults [2]. The consequence of a fall can profoundly affect the physical and psychological well-being of a person, leading to a fear of falls, decreased mobility with a loss of independence, social isolation, earlier institutionalization, and a lessened quality of life [3]. The 
use of a mobility aid is a standard treatment option for balance and gait impairments, yet mobility aids in dementia are associated with a three-fold increase in the odds of falls $[4,5]$. There is a desperate need to find successful interventions to prevent falls for the more than 25 to 37.5 million people globally currently living with $\mathrm{AD}[6]$.

Walking involves planning and navigating through environments to manage obstacles, changes in terrain, and unexpected perturbations without becoming unsteady and losing balance [7]. As such, gait is a cognitively demanding task that requires higher order cognitive functions for even the regulation of routine walking [8]. Executive function, a collection of cognitive processes such as attention and memory, is critical to the planning and modification of walking according to sensory information and environmental factors (e.g., obstacles) [9]. Importantly, executive function impairment is associated with an increased risk of falls [10]. In AD, executive function is impaired early [11], progressively deteriorates and has been found to be associated with an unstable gait performance [12].

Most of our everyday activities involve the completion of two tasks at once, known as dual-tasking [9]. Attention is considered to have a finite capacity and if the performance of two tasks simultaneously exceeds the cognitive capacity of the individual then performance on one or both concurrent tasks will deteriorate [14]. In those with functional limitations (e.g., a walking impairment), a greater amount of attentional demand is needed when walking in order to accommodate for a less stable system [8]. The change in performance from the single-task to a combined task, is known as task cost, and is a measure of the cognitive demands imposed by the tasks [14]. Cognitive demands will vary with task novelty and task complexity, with an increased cognitive demand being associated with an increased falls risk $[14,15]$. Dual-task testing enables the study of the inter-relationship between cognition and mobility. Importantly, when people with $\mathrm{AD}$ walk and perform a concurrent cognitive task, they exhibit a deterioration in walking quality as demonstrated by slower walking and greater stride time variability than walking alone [16].

Provision of a mobility aid (e.g., a cane or walker) to those displaying balance and gait problems can facilitate independent and safe ambulation during activities of daily living [17]. A mobility aid improves stability by increasing the base-of-support and allowing for sensory feedback to be gathered through the upper body [17]. Different types of mobility aids can provide varying levels of support from a small (e.g., a single-point cane) to a large (e.g., wheeled walker) amount. The aim is to provide a gait aid that delivers the level of support required by the individual to optimize gait, but not too much support that it may limit function. The optimal and safe use of a single-point cane requires appropriate sequencing and coordination of the gait aid and leg [18], and use of the aid when turning or negotiating obstacles. Although not often considered as such, the use of a mobility aid is a complex motor task that can result in an increased cognitive load [17].

Impairment in executive function can result in a reduced ability to successfully ambulate in attentiondemanding situations. People with mild to moderate $\mathrm{AD}$ learning to use a 4-wheeled walker had slower performance times and an increased number of steps compared to healthy controls manoeuvring around obstacles, yet there was no difference between groups when walking in a straight path with the aid [19]. Learning new tasks requires increased attention until users become more experienced. Walking with a single-point cane has been found to increase cognitive task demands in older adults experienced with using a cane [20]. The effect on spatial-temporal gait parameters while learning to use a single-point cane in people with mild to moderate $\mathrm{AD}$ has not been studied. The aim of this study was to evaluate in cognitively-healthy older adults and people with mild to moderate $\mathrm{AD}: 1$ ) the changes in spatial-temporal gait parameters of velocity and stride time variability, and 2) the gait and cognitive task cost of newly learning to use a single-point cane during dual-task testing. It was hypothesized that using a single-point cane would result in gait instability, more pronounced with complex walking paths and while multi-tasking in the $\mathrm{AD}$ group than the cognitively healthy older adults.

\section{METHODS}

\section{Participants}

Study participants comprised two groups, cognitively-healthy adults and adults diagnosed with AD. Participants with AD were recruited from a local day program, where referral is based on a confirmed diagnosis of dementia by a geriatrician according to the criteria of the National Institute of Neurologic and Communicative Disorders and Stroke-AD and Related Disorders Association (NINCDSARDRA) [21]. The controls were recruited through e-newsletters from a community fitness program. This study was approved by the Health Sciences 
Ethics Review Board of the University of Western Ontario. Cognitively normal participants provided written informed consent, whereas participants with $\mathrm{AD}$ either provided written informed consent themselves or a substitute decision maker provided this consent and the participant provided assent to participate in the study. Data collection took place between March 2017 and May 2018.

Inclusion criteria for the AD group were a diagnosis of mild to moderate AD (Mini-Mental State Examination [22] between 11-20 indicated moderate severity and scores between 21-24 indicated mild severity of $\mathrm{AD}$ [23]), aged 50 years and older, able to walk independently for 30 meters without the use of a mobility aid or the assistance of another person. Inclusion criteria for the controls were being aged 50 years and older, able to walk 30 meters unassisted by a mobility aid or assistance of another person, no subjective cognitive complaints, and a score on the MMSE greater than 24. At the time of data collection, participants did not rely on or need the use of mobility aids for ambulation indoors or outdoors. Exclusion criteria for both groups were an inability to understand verbal instructions given in English, any neurological disorder with motor deficits (e.g., Parkinson's disease, stroke), or severe musculoskeletal disorders that impact walking.

\section{Outcome measures}

Participants in both groups completed the same study procedures. Participants or the substitute decision maker provided socio-demographic and medical information, including age, gender, years of formal education, co-morbidities, prescription medications, physical activity levels (assessed by self-report: vigorous, engages in structured exercise program for 30 minutes three times a week; moderate, engages in physical activity at least three times a week; sedentary physical activity less than three times a week), visual acuity and basic and instrumental activities of daily living, as per the Lawton-Brody Instrumental Activities of Daily Living (IADL) and Basic Activities of Daily Living (BADL) scales [24]. Participants in both groups also completed the IconographicalFalls Efficacy Scale (ICON-FES), which has been validated in older adults with and without cognitive impairment $[25,26]$.

\section{Single-task cognitive assessment}

The cognitive task of serial subtractions by ones, starting at 100, was performed by each participant in a seated position prior to the gait tests. Time to complete 10 subtractions was recorded to the nearest 100 th of a second with a stop watch. The total number of responses and number of correct responses given were recorded.

\section{Gait assessment}

Gait performance was assessed using tri-axial accelerometers (Locomotion Evaluation and Gait System, LEGSys ${ }^{\mathrm{TM}}$, BioSensics, Cambridge, MA). Two sensors were used, one worn on each of the lower limbs in the frontal plane. Gait parameters of interest were velocity and stride time variability [27]. Variability was quantified using the coefficient of variation, the ratio of the standard deviation of the mean stride time to the mean stride time multiplied by $100 \%$. All gait testing was performed at a self-selected usual walking speed. Each participant performed a practice trial of each walking task and during testing the person completed two trials that were averaged for analysis. Between trials participants had the ability to rest before proceeding to the next trial/condition.

Gait was evaluated on two path configurations: a straight path (SP) of 6 meters and the Figure of Eight Walking Test (F8) [28] under three tasks for a total of 6 test conditions: 1) single-task (ST) in which participants only walked each path (SP_ST, F8_ST); 2) dual-task (DT), in which participants walked each path while using a single-point cane (SP_DT, F8_DT); and 3) multi-task (MT), in which participants walked each path while using a single-point cane and counting backwards from 100 by $1 \mathrm{~s}$ (SP_MT, F8_MT). Number and accuracy of the responses on the secondary cognitive task were recorded during the multi-task test conditions. There was no instruction to prioritize the gait or cognitive task during multi-task testing.

Participants were provided with a single-point straight cane that was sized to each person by the research assistant (i.e., height of the top of the cane handle was adjusted to be level with wrist crease with the arm hanging by the participant's side when standing erect). The cane was to be used in the person's dominant hand and each person was given instructions on how to appropriately use the cane while walking, repeating instructions as required for people in both groups. Participants were allotted 5 minutes to practice walking around the room with the gait aid before gait testing commenced. Participants were observed and provided feedback to ensure that the equipment was being used correctly prior to starting testing. 


\section{Data analysis}

The first objective was evaluated through a comparison of the gait parameters of velocity and stride time variability across walking conditions and between groups using a 2-way repeated measures ANOVA, adjusted for age. The factors were group (older adult and $\mathrm{AD}$ ) as the between groups variable, and walking condition (the six test conditions) as the within-group variable. Where appropriate for control of multiple comparison bias, per-comparison was undertaken using a Bonferroni correction. Cohen's d effect size (ES) was calculated to quantify the magnitude of the difference between the two groups:

$$
d=\frac{M_{\text {group } 1}-M_{\text {group } 2}}{S D_{\text {pooled }}}
$$

Benchmark values for ES to estimate the magnitude of the effect and classified were: trivial $(<0.20)$, small $(0.20$ to $<0.50)$, moderate $(0.50$ to $<0.80)$, or large $(>0.80)$ [29].

To address the second objective, two new variables were calculated to quantify the task cost for each component of the combined activity, cognition and gait, for the dual-task and multi-task test conditions. Task cost for gait was calculated as the percentage change in velocity for the single-task of walking to the dual-task and multi-task conditions:

$$
\left[\frac{S P_{S T}-D T \text { or } M T}{S P_{S T}}\right] \times(100)(-1)
$$

Task cost for cognitive performance was determined by first calculating the correct response rate (CRR) for the single-task cognitive test and multitask tests as: (Response rate per second $\mathrm{X}$ percent correct). CRR accounts for speed and accuracy of responses given [30]. DTCcog was calculated as:

$$
\begin{aligned}
& {\left[\frac{\text { CRRserialsubtractionsseated }- \text { CRRserialsubtractionswalking }}{\text { CRRserialsubtractionsseated }}\right]} \\
& \quad \times(100)(-1)
\end{aligned}
$$

The interpretation of the task cost value is the same for both gait and cognition. A negative value indicates poorer performance under the dual-task or multi-task conditions (e.g., slower velocity under the dual-task condition). A positive value indicates better performance under dual-task or multi-task conditions (e.g., faster velocity or greater number of responses or greater accuracy of responses). A comparison of gait task cost for velocity between the groups using a similar the ANOVA procedure described for the first objective.

A performance-resource operating characteristic (POC) graph was created by plotting cognitive task cost (x-axis) versus gait task cost (y-axis) for the multi-task test conditions to demonstrate the trade-off between the gait and cognitive tasks [14]. Performance will fall into one of four quadrants: 1) upper left - improvement of gait with worsening of cognitive task, 2) upper right - improvement of gait with improvement of cognitive task, 3) lower left - worsening of gait with worsening of cognitive task, and 4) lower right - worsening of gait with improvement of cognitive task. Performance that falls on the axes at $0 \%$ task cost for gait and cognition indicates no change in performance between single- and dual-task conditions [31]. A diagonal line cuts through quadrants 2 and 3 , this line indicates a $1: 1$ trade-off during dual-task performance; to the left of this line gait is prioritized and the cognitive task is prioritized to the right of the line [31].

Sample size calculation was based on our previous research in people with dementia [19], and suggested that a sample size of 25 participants is needed for a power of $80 \%$ with $\alpha=0.05$ to detect a $10 \%$ difference in dual-task cost.

\section{RESULTS}

Twenty-five people with AD were enrolled in the study, but three withdrew for health reasons before data collection and five were unable to complete the full gait testing protocol. Seventeen participants with $\mathrm{AD}$ (age $=82.1 \pm 5.6$ years) and 25 controls (age $=70.8 \pm 14.1$ years) who participated in the study had full data. Participants with AD were older, had less education, and had lower instrumental activities of daily living scores. As anticipated, individuals with AD had lower scores on all cognitive measures. Characteristics of the participants are presented in Table 1.

Gait velocity results suggested a non-significant interaction between group and test condition, but statistically significant main effects for group $(p=0.001)$ and test condition $(p<0.001)$. This is presented graphically in Fig. 1. (See Supplementary Table 1 and Table 2 for post hoc comparisons). Participants with $\mathrm{AD}$ demonstrated significantly slower gait as task complexity increased for all Figure of 8 test conditions, but no difference between simple path without and with the cane $(p=0.068)$. Similarly, 
Table 1

\begin{tabular}{lccc}
\multicolumn{4}{c}{$\begin{array}{c}\text { Demographic and clinical characteristics of the sample of cognitively healthy adults and adults with } \\
\text { mild to moderate Alzheimer's disease }\end{array}$} \\
\hline Variable & $\begin{array}{c}\text { Controls } \\
(n=25)\end{array}$ & $\begin{array}{c}\text { Alzheimer's } \\
\text { disease }(n=17)\end{array}$ & $p$ \\
& $70.8 \pm 14.1$ & $82.1 \pm 5.6$ & 0.001 \\
Age (y) & $19(76)$ & $6(35)$ & 0.007 \\
Sex $(n, \%$ female) & $26.8 \pm 6.8$ & $26.1 \pm 5.2$ & 0.732 \\
Body Mass Index $\left(\mathrm{kg} / \mathrm{m}^{2}\right)$ & $16.4 \pm 3.8$ & $12.0 \pm 3.7$ & 0.003 \\
Education (y) & $11.8 \pm 3.2$ & $14.8 \pm 4.9$ & 0.036 \\
Iconographical Falls Efficacy Scale & $8.0 \pm 0.0$ & $2.5 \pm 1.9$ & $<0.001$ \\
Instrumental Activities of Daily Living & $6.0 \pm 0.0$ & $5.8 \pm 0.4$ & 0.083 \\
Basic Activities of Daily Living & $0.14 \pm 0.12$ & $0.24 \pm 0.26$ & 0.163 \\
High Contrast Acuity (Minimum angle of resolution) & $0.34 \pm 0.15$ & $0.56 \pm 0.30$ & 0.011 \\
Low Contrast Acuity (Minimum angle of resolution) & $4(16 \%)$ & $3(17.6 \%)$ & 0.973 \\
History of falls in past 12 months $(n, \%)$ & & 0.094 \\
Physical Activity ( $n, \%)$ : & $2(8.0 \%)$ & $3(17.6 \%)$ & \\
Sedentary & $9(36.0 \%)$ & $9(52.9 \%)$ & \\
Moderate & $14(56.0 \%)$ & $5(29.4 \%)$ & $<0.001$ \\
Vigorous & $29.0 \pm 1.9$ & $19.6 \pm 5.4$ & 0.001 \\
Mini-Mental State Examination & $2.40 \pm 2.21$ & $5.47 \pm 3.13$ & 0.480 \\
Number of Prescription Medications & $1.96 \pm 2.01$ & $2.35 \pm 1.27$ & \\
Number of Comorbidities & & & 0.824 \\
Summary of comorbidities (n, \% yes) & $8(32.0 \%)$ & $6(42.9 \%)$ & 0.021 \\
Hypertension & $1(4.0 \%)$ & $5(29.4 \%)$ & 0.482 \\
Myocardial Infarction & $5(29.4 \%)$ & $5(20.0 \%)$ & 0.049 \\
Cataract & $5(20.0 \%)$ & $0(0 \%)$ & 0.391 \\
Macular Degeneration & $13(76.5 \%)$ & $16(64.0 \%)$ &
\end{tabular}

controls tended to show slower gait velocities as the complexity of the walking task increased, but demonstrated no statistically significant difference between straight path multi-tasking and Figure of 8 singletask $(p=0.587)$. This is presented in Supplementary Table 2. Gait velocity was significantly slower in the $\mathrm{AD}$ group compared to the older adults for all test conditions $(p<0.001)$ and all between comparisons had large effect sizes.

Stride time variability demonstrated a statistically significant interaction between group and test $(p<0.001)$ and the main effect of group $(p=0.003)$ (Fig. 2). The group of adults with AD had greater stride time variability for straight path multi-tasking $(p=0.03)$ and for all of the Figure of 8 test conditions: walking only $(p=0.003)$, walking with cane $(p=0.004)$ and multi-tasking $(p<0.001)$. Between group comparison found a statistically difference between people with $\mathrm{AD}$ and controls only in the test condition of Figure of 8 multitasking $(p<0.001)$.

The results of the analyses of gait task cost for velocity are shown in Table 2 . There were no other statistically significant findings demonstrated for gait task cost, specifically test condition $(p=0.147)$, group $(p=0.587)$, interaction condition $\mathrm{X}$ group (0.131). The gait velocity task costs for using a cane only did not vary between the two groups walking in a straight path (AD was $-8.4 \pm 10.9 \%$ and older adults were $-14.9 \pm 15.9 \%$ ) and walking in a Figure of 8 ( $\mathrm{AD}$ was $-5.3 \pm 10.5 \%$ and older adults were $-8.2 \pm 12.0 \%)$.

The cognitive task costs were significant for an interaction between task and group $(p=0.003)$, but not for the main effects of test condition $(p=0.867)$ and group $(p=0.292)$ (Table 2) Cognitive task costs were stable for the controls, though values for the people with $\mathrm{AD}$ decreased in the multitask situation. In terms of cognitive performance, both groups were observed to have similar accuracy at baseline (AD was $97.65 \pm 0.06 \%$ and older adults were $99.20 \pm 0.04 \%$; $p=0.603$ ). However, and as expected, accuracy of cognitive task responses differed when walking in a straight path (AD was $97.68 \pm 0.04 \%$ and older adults were $100.00 \pm .00 \%$; $p=0.030)$ and Figure of 8 (AD was $88.01 \pm 0.13 \%$ and older adults were $94.36 \pm 0.10 \% ; p<0.001)$. There were effects also with the response rate. At baseline, people with $\mathrm{AD}$ had a lower response rate at $0.90 \pm 0.42$ words per second compared to the controls at $1.54 \pm 0.43$ words per second $(p<0.001)$. Response rate decreased in both groups during the multitask walking conditions, performance was lower in people with AD. Specifically in the straight path multitask, the people with $\mathrm{AD}$ had $0.74 \pm 0.24$ 


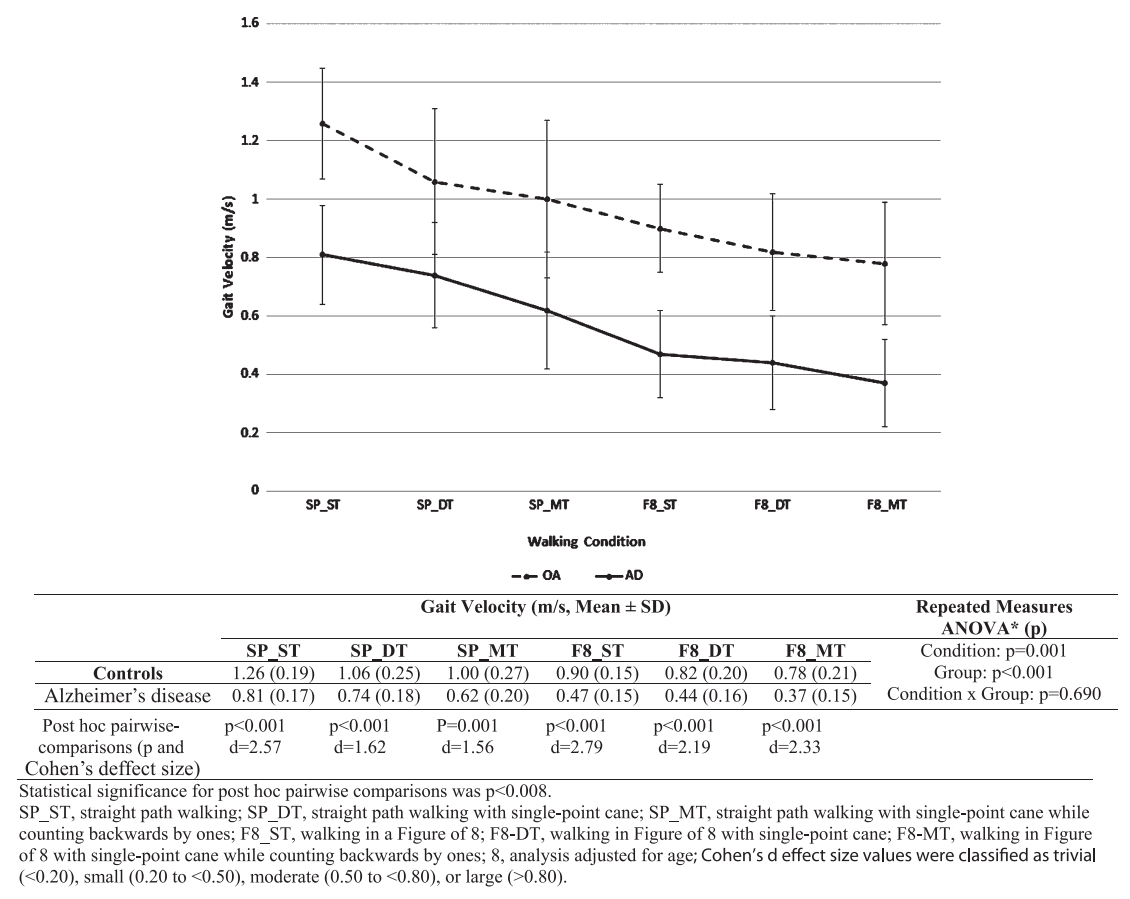

Fig. 1. Gait velocity for adults and adults with mild to moderate Alzheimer's disease while learning to use a single-point cane under straight and Figure of 8 path configuration.

words per second and the controls had $0.97 \pm 0.29$ $(p=0.042)$ and in the figure of 8 multitask the people with AD had $0.65 \pm 0.44$ words per second and the controls had $1.00 \pm 0.35(p=0.021)$.

The POC graph (Fig. 3) demonstrated mutual interference for the gait and cognitive activities in the straight path and Figure of 8 path, performance on cognition and gait deteriorated in the multi-task test condition for both groups. In the straight path multitask condition, $65 \%$ (11/17) of people with AD and $68 \%(17 / 25)$ of controls prioritized the gait task over the cognitive performance. In the Figure of 8 pathway, $47 \%(8 / 17)$ and $68 \%$ (17/25) controls prioritized gait over the cognitive task.

\section{DISCUSSION}

This study has demonstrated that cognitively healthy adults and people with mild to moderate $\mathrm{AD}$ learning to use a single-point cane experience a decrease in their gait performance and an increase in cognitive demands. People with AD walked slower than the controls in all test conditions. The more complex walking configuration of the Figure of 8 produced a greater degree of change in the gait than the straight path. Task prioritization in the multi-task scenarios demonstrated a mutual interference for gait and cognitive tasks for both groups with a decrease in gait and cognitive performance, but cognitive performance had a statistically significant decrease for the $\mathrm{AD}$ group in the most complex pathway. This is the first study to evaluate changes in gait parameters and cognitive cost in healthy older adults and adults with AD learning to use a cane.

The literature supports how the use of a mobility aid (e.g., a cane or walker) for people displaying balance and gait problems can facilitate independent and safe ambulation, and activities of daily living [17]. Hardi et al. [18] demonstrated older adults who are experienced users of a single-point cane had better walking performance when using their cane compared to unassisted walking. In contrast, our study demonstrated that learning to use a cane resulted in a deterioration in gait velocity, which was greater in adults with mild to moderate AD in multi-tasking in a straight path and in all complex path testing. The effect of a training program to use a single-point cane is warranted to establish whether a change in motor activity with practice can be achieved.

We have also demonstrated that cognitive demands increase with learning to use a cane, consistent with competition for available attentional resources for neuromotor control while performing a novel motor activity [14]. The use of a cane has unique demands 


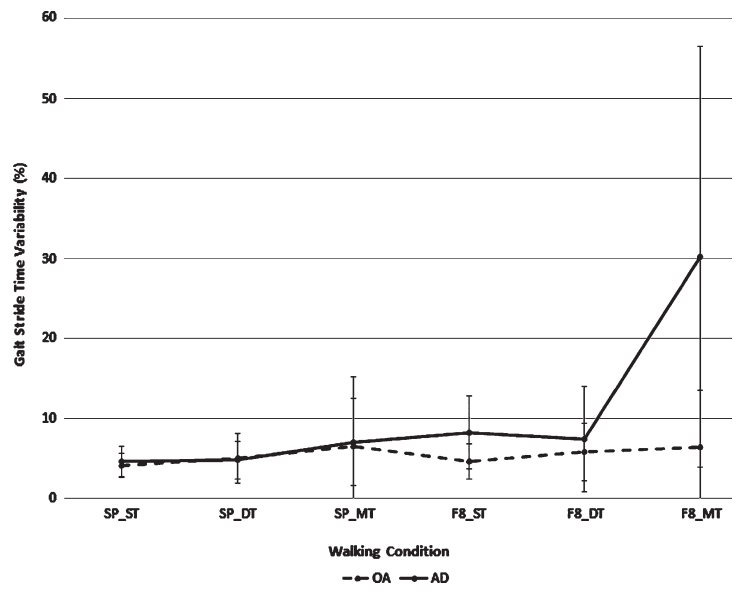

\begin{tabular}{|c|c|c|c|c|c|c|c|}
\hline & \multicolumn{6}{|c|}{ Gait Stride Time Variability $(\%$, Mean \pm SD) } & \multirow{3}{*}{$\begin{array}{c}\text { 2-way Repeated Measures } \\
\text { ANOVA* (p) } \\
\text { Condition: } \mathrm{p}=0.259 \\
\text { Group: } \mathrm{p}=0.003\end{array}$} \\
\hline & SP_ST & SP_DT & SP_MT & $\begin{array}{c}\text { F8_ST } \\
462(2.21)\end{array}$ & F8_DT & F8_MT & \\
\hline Controls & $4.13(1.51)$ & $5.01(3.10)$ & $6.50(8.78)$ & $4.62(2.21)$ & $5.83(3.57)$ & $6.42(7.13)$ & \\
\hline Alzheimer's disease & $4.68(1.90)$ & $4.80(2.38)$ & $7.07(5.45)$ & $8.25(4.54)$ & $7.43(6.63)$ & $30.24(26.30)$ & Condition $\mathrm{x}$ Group: $\mathrm{p}<0.001$ \\
\hline $\begin{array}{l}\text { Post hoc pairwise- } \\
\text { comparisons (p and } \\
\text { Cohen's deffect size) }\end{array}$ & $\begin{array}{c}\mathrm{p}=0.390 \\
\mathrm{~d}=0.32\end{array}$ & $\begin{array}{c}\mathrm{p}=0.691 \\
\mathrm{~d}=0.08\end{array}$ & $\begin{array}{c}\mathrm{p}=0.667 \\
\mathrm{~d}=0.08\end{array}$ & $\begin{array}{c}\mathrm{p}=0.009 \\
\mathrm{~d}=1.08\end{array}$ & $\begin{array}{c}\mathrm{p}=0.461 \\
\mathrm{~d}=0.31\end{array}$ & $\begin{array}{c}\mathrm{p}<0.001 \\
\mathrm{~d}=1.42\end{array}$ & \\
\hline
\end{tabular}

Fig. 2. Stride time variability for older adults and adults with mild to moderate Alzheimer's disease while learning to use a single-point cane under straight and Figure of 8 path configuration.

that can challenge cognitive function due to the asymmetric use of one aid that requires the person to coordinate the motor sequencing of the cane and the opposite leg movement, advancing the cane appropriately without contact with the ipsilateral leg or obstacles in the environment, appropriate placement of the cane for support and ensuring ongoing forward movement of the body [17].

Additionally, we were able to demonstrate that the majority of people in both groups self-selected to pri- oritize the gait task over the cognitive task, a posture first strategy [8]. Yet, more participants with AD prioritized the cognitive task over the mobility task in both path configurations demonstrating a posture second strategy that may also lead to instability and falls [8]. The cognitive task performance was impacted by both changes in accuracy of responses and the response rate, the people with $\mathrm{AD}$ having greater decrements in both areas than the controls. The use of a summary value for cognitive performance does

Table 2

Task costs for gait and cognition for cognitively healthy older adults and adults with Alzheimer's disease while walking with a cane and walking with a cane while counting backwards by ones

\begin{tabular}{|c|c|c|c|c|c|}
\hline & \multicolumn{4}{|c|}{ Mean \pm SD } & \multirow{2}{*}{$\begin{array}{c}\text { 2-way Repeated Measures } \\
\text { ANOVA* }^{*}(p)\end{array}$} \\
\hline & SP_DT & SP_MT & F8_DT & F8_MT & \\
\hline \multicolumn{6}{|c|}{ A. Task cost for gait $(\%)$} \\
\hline Alzheimer's disease & $-8.4(10.9)$ & $-24.6(13.5)$ & $-5.3(10.5)$ & $-21.4(20.9)$ & $\begin{array}{c}\text { Condition: } p=0.147 \\
\text { Group: } p=0.587\end{array}$ \\
\hline Controls & $-14.9(15.9)$ & $-22.7(17.4)$ & $-8.2(12.0)$ & $-13.4(13.1)$ & Condition x Group: $p=0.131$ \\
\hline \multicolumn{6}{|c|}{ B. Task cost for cognition (\%) } \\
\hline Alzheimer's disease & & $-9.6(30.8)$ & & $-35.7(28.0)$ & $\begin{array}{c}\text { Condition: } p=0.867 \\
\text { Group: } p=0.292\end{array}$ \\
\hline Controls & & $-33.2(23.6)$ & & $-33.0(23.5)$ & Condition x Group: $p=0.003$ \\
\hline
\end{tabular}

SP_DT, straight path and walking with cane; SP_MT, straight path and walking with a cane while counting backwards by ones; F8_DT, figure of 8 path and walking with a cane; F8_MT, figure of 8 path and walking with a cane while counting backwards by ones; *, analysis adjusted for age. 


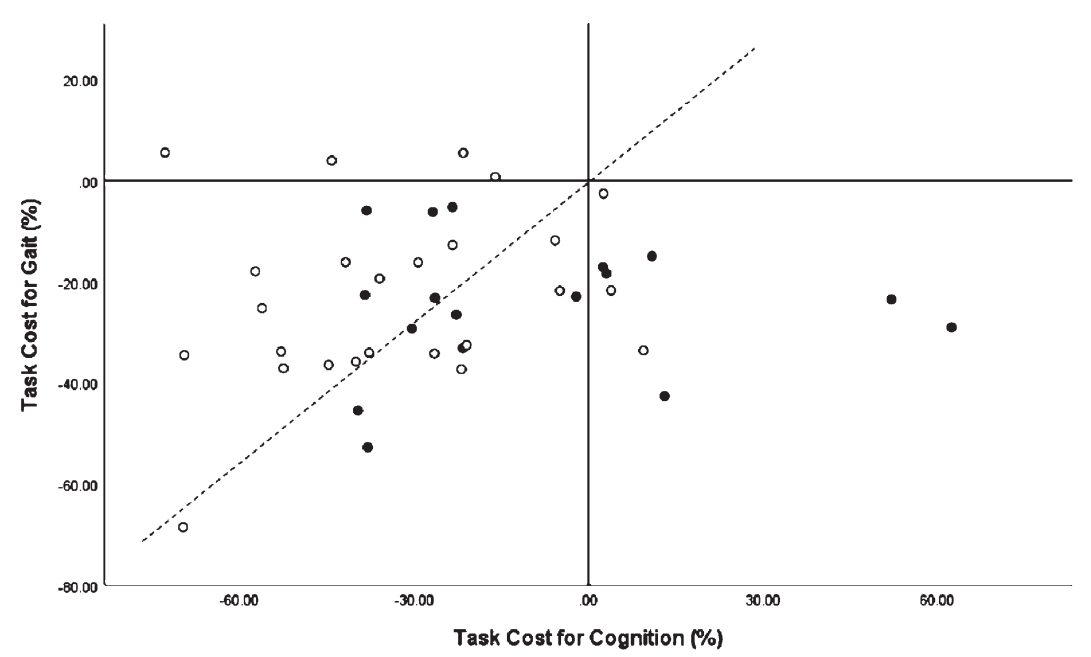

a) Straight path with multi-tasking

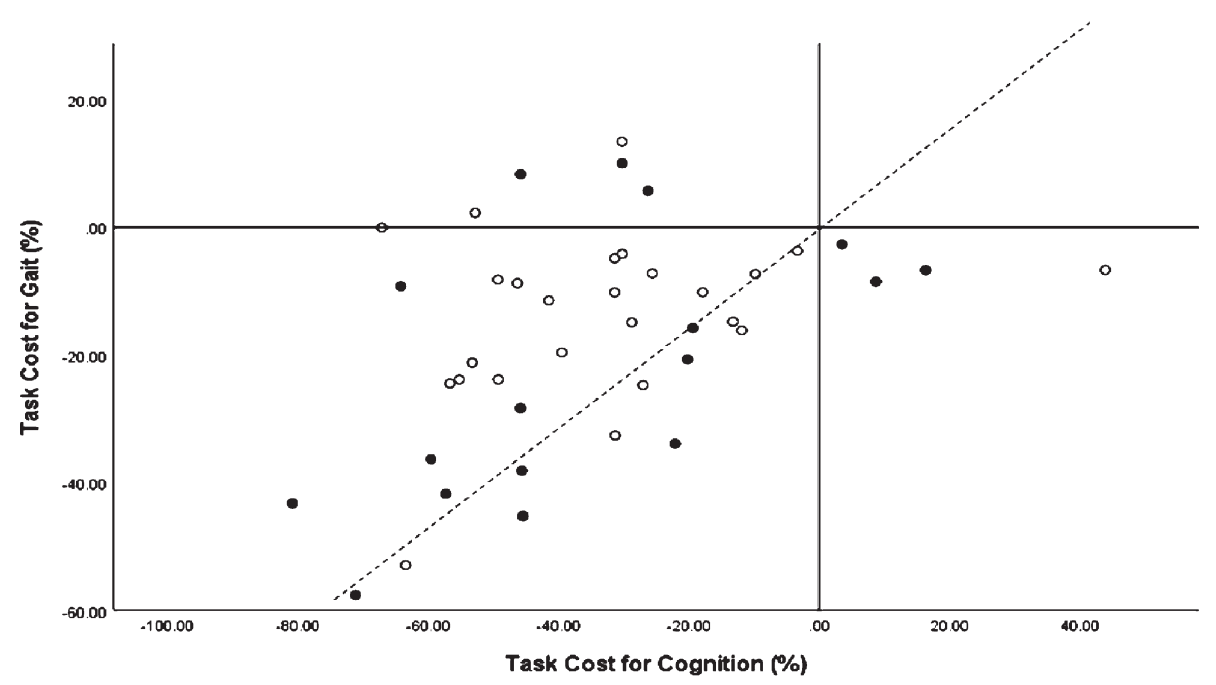

b) Figure of 8 with multi-tasking

Fig. 3. Performance-resource operating characteristic graph for demonstration of between task trade-offs of gait and cognitive tasks during multi-task gait testing (walking while using a cane and counting backwards by ones) in cognitively healthy older adults (o) and people with mild to moderate Alzheimer's disease (•).

not allow for transparency of how the components of the performance of the task were impacted and therefore the explicit reporting of both accuracy and response rate in very important information. Mobility aids can be associated with an increased fall risk, especially in people with dementia [1]. Control of postural stability and the normal regulation of gait requires ongoing cognitive processing $[8,32]$. The prescription of mobility aids for people with dementia may occur at a point in the disease when available cognitive resources are limited. As a consequence, contrary to the intended benefits, people with demen- tia may experience instability, falls, and fractures while using a mobility aid. As such, there may be a need for clinicians who are considering use of a cane for people with AD to evaluate the effect on stability by trialing use before prescribing the gait aid. It may be that use of a cane will be beneficial for some people with $\mathrm{AD}$ and be detrimental for others.

The underlying mechanisms for an increased falls risk in adults with dementia are not well understood, though it is known that mobility aids are associated with an increased risk [1]. The present changes 
found in gait contribute to our understanding of possible factors underlying the increased risk. More research is required to explore issues related to the uptake and safe use of mobility aids that may be impacted by cognitive impairment through a lack of self-awareness or incomplete learning to use the equipment. The unsafe use of canes is a common finding in cognitively healthy community-dwelling older adults and includes lack of training from a health care professional to use the equipment, incorrect sizing, inability to maintain the proper reciprocal gait pattern, and inappropriate posture [33]. Cerebral amyloid-beta has been found to impair motor sequencing in dual-task gait testing in older adults [34], so the use of tests to evaluate motor sequencing ability may be a valuable screen for rehabilitation professionals to use in assessment and training for use of mobility aids. There is more work required before this work can be transferred into clinical practice for assessment and training purposes for everyday use and its potential implications to falls prevention strategies.

This study has several limitations that should be considered in the interpretation of the findings. We had a priori planned to recruit 25 people with $\mathrm{AD}$, but unfortunately $32 \%$ of the people enrolled in the study had to withdraw or were unable to complete the full gait testing protocol. A post hoc sample size calculation established we had the power to be able to detect a $15 \%$ difference in task cost, as we did observe statistically significant differences between groups these values likely represent a conservative estimate of effect. The sample may have lacked the power to be able to find other differences in the temporal and spatial gait parameters. Our sample is not generalizable to all people with $\mathrm{AD}$ due to variations that are expected in cognition based on disease severity and our participants with $\mathrm{AD}$ were all recruited from a specialty day hospital program for people with dementia. In addition, there were demographic differences between the AD and control group noted, thus findings may not solely be attributable to cognitive status alone. Also, this study only evaluated single-point canes and there are other types of canes (e.g., four-point canes) that may lead to different changes in gait. There are several strengths we would like to highlight including the assessment of spatialtemporal gait parameters in people who do not use and are not in need of a mobility aid that allowed the evaluation of new learning effects. We also assessed task costs related to both gait and cognition, the evaluation of both allows for determination of intrinsic task prioritization for the individual and use of a posture first or posture second strategy in challenging situations.

\section{Conclusions}

Learning to walk using a single-point cane is a cognitively demanding task for cognitively healthy older adults and adults with mild to moderate dementia, though the magnitude of effect is greater for people with AD. Individuals with AD had statistically significant increases in gait variability in more complex paths needing to maneuver around obstacles and when multitasking walking in a straight line. Multi-tasking, walking with a cane and counting by ones, confers a greater cognitive demand and greater deterioration on gait quality, and reflects real world tasks for people using a cane. More research is needed to evaluate the effect of a training program on these effects to determine capacity and timing for improvements to occur with practice of the activity.

\section{ACKNOWLEDGMENTS}

The authors thank Karen Johnson, Director of Alzheimer Outreach Services of McCormick Home; Steve Crawford, CEO, McCormick Care Group and the staff and clients at the Alzheimer Outreach Services day program for their hospitality, assistance in organizing this project and participation in the data collection process. This study was funded by the Alzheimer's Association (AARG-16-440671) and had no involvement in the conduct of the study.

Authors' disclosures available online (https:// www.j-alz.com/manuscript-disclosures/18-1169r2).

\section{SUPPLEMENTARY MATERIAL}

The supplementary material is available in the electronic version of this article: http://dx.doi.org/ 10.3233/JAD-181169.

\section{REFERENCES}

[1] Fernando E, Fraser M, Hendriksen J, Kim CH, Muir-Hunter SW (2017) Risk factors associated with falls in older adults with dementia: A systematic review. Physiother Can 69, 161-170.

[2] Allan LM, Ballard CG, Rowan EN, Kenny RA (2009) Incidence and prediction of falls in dementia: a prospective study in older people. PLoS One 4, e5521. 
[3] Stinchcombe A, Kuran N, Powell S (2014) Seniors' falls in Canada: Second report.

[4] Pellfolk T, Gustafsson T, Gustafson Y, Karlsson S (2009) Risk factors for falls among residents with dementia living in group dwellings. Int Psychogeriatrics 21, 187.

[5] Panel on Prevention of Falls in Older Persons, American Geriatrics Society and British Geriatrics Society (2011) Summary of the updated American Geriatrics Society/British Geriatrics Society clinical practice guideline for prevention of falls in older persons. J Am Geriatr Soc 59, 148-157.

[6] Prince M, Comas-Herrera A, Knapp M, Guerchet M, Karagiannidou M (2016) World Alzheimer Report 2016. Improving Healthcare for People Living with Dementia: Coverage, Quality and Costs Now and in the Future. Alzheimer's Disease International, London, UK.

[7] Frank J (2003) Balance and mobility challenges in older adults:Implications for preserving community mobility. Am J Prev Med 25, 157-163.

[8] Woollacott M, Shumway-Cook A (2002) Attention and the control of posture and gait: A review of an emerging area of research. Gait Posture 16, 1-14.

[9] Yogev-Seligmann G, Hausdorff JM, Giladi N (2008) The role of executive function and attention in gait. Mov Disord 23, 329-342.

[10] Muir S, Gopaul K, Montero Odasso MM (2012) The role of cognitive impairment in fall risk among older adults: a systematic review and meta-analysis. Age Ageing 41, 299-308.

[11] Perry RJ (1999) Attention and executive deficits in Alzheimer's disease: A critical review. Brain 122, 383-404.

[12] Allan LM, Ballard CG, Burn DJ, Kenny RA (2005) Prevalence and severity of gait disorders in Alzheimer's and non-Alzheimer's dementias. J Am Geriatr Soc 53, 1681-1687.

[13] Association A (2018) 2018 Alzheimer's disease facts and figures. Alzheimers Dement 14, 367-429.

[14] McIsaac TL, Lamberg EM, Muratori LM (2015) Building a framework for a dual task taxonomy. Biomed Res Int 2015, 591475.

[15] Muir-Hunter SW, Wittwer JE (2015) Dual-task testing to predict falls in community-dwelling older adults: a systematic review. Physiotherapy 102, 29-40.

[16] Muir SW, Speechley M, Wells J, Borrie M, Gopaul K, Montero-Odasso M (2012) Gait assessment in mild cognitive impairment and Alzheimer's disease: The effect of dual-task challenges across the cognitive spectrum. Gait Posture 35, 96-100.

[17] Bateni H, Maki BE (2005) Assistive devices for balance and mobility: Benefits, demands, and adverse consequences. Arch Phys Med Rehabil 86, 134-145.

[18] Härdi I, Bridenbaugh SA, Gschwind YJ, Kressig RW (2014) The effect of three different types of walking aids on spatiotemporal gait parameters in community-dwelling older adults. Aging Clin Exp Res 26, 221-228.

[19] Muir-Hunter S., Montero-Odasso M, Montero Odasso M (2017) The attentional demands of ambulating with an assistive device in older adults with Alzheimer's disease. Gait Posture 54, 202-208.

[20] Wellmon R, Pezzillo K, Eichhorn G, Lockhart W, Morris J (2006) Changes in dual-task voice reaction time among elders who use assistive devices. J Geriatr Phys Ther 29, 74-80.
[21] Albert MS, DeKosky ST, Dickson D, Dubois B, Feldman HH, Fox NC, Gamst A, Holtzman DM, Jagust WJ, Petersen RC, Snyder PJ, Carrillo MC, Thies B, Phelps CH, McKhann GM, Knopman DS, Chertkow H, Hyman BT, Jack CR, Kawas CH, Klunk WE, Koroshetz WJ, Manly JJ, Mayeux R, Mohs RC, Morris JC, Rossor MN, Scheltens P, Carrillo MC, Thies B, Weintraub S, Phelps CH (2011) The diagnosis of mild cognitive impairment due to Alzheimer's disease: Recommendations from the National Institute on Aging-Alzheimer's Association workgroups on diagnostic guidelines for Alzheimer's disease. Alzheimers Dement 7, 270-279.

[22] Folstein MF, Folstein SE, McHugh PR (1975) Mini-Mental State: A practical method for grading the state of patients for the clinician. J Psychiatr Res 12, 189-198.

[23] Perneczky R, Wagenpfeil S, Komossa K, Grimmer T, Diehl J, Kurz A (2006) Mapping scores onto stages: Mini-mental state examination and clinical dementia rating. Am J Geriatr Psychiatry 14, 139-144.

[24] Lawton MP, Brody EM (1969) Assessment of older people: self-maintaining and instrumental activities of daily living. Gerontologist 9, 179-186.

[25] Delbaere K, T. Smith S, Lord SR (2011) Development and initial validation of the iconographical falls efficacy scale. J Gerontol A Biol Sci Med Sci. 66A, 674-680.

[26] Delbaere K, Close JCT, Taylor M, Wesson J, Lord SR (2013) Validation of the iconographical falls efficacy scale in cognitively impaired older people. J Gerontol A Biol Sci Med Sci 68, 1098-1102.

[27] Hollman JH, Kovash FM, Kubik JJ, Linbo RA (2007) Age-related differences in spatiotemporal markers of gait stability during dual task walking. Gait Posture 26, 113-119.

[28] Hess RJ, Brach JS, Piva SR, VanSwearingen JM (2010) Walking skill can be assessed in older adults: validity of the Figure-of- 8 Walk Test. Phys Ther 90, 89-99.

[29] Cohen J (1988) Statistical power analysis for the behavioral sciences. Lawrence Erlbaum Associates.

[30] Hall CD, Echt K V, Wolf SL, Rogers WA (2011) Cognitive and motor mechanisms underlying older adults' ability to divide attention while walking. Phys Ther 91, 1039-1050.

[31] Frengopoulos C, Payne MW, Holmes JD, Viana R, Hunter SW (2018) Comparing the effects of dual-task gait testing in new and established ambulators with lower extremity amputations. $P M \& R$ 10, 1012-1019.

[32] Montero-Odasso M, Verghese J, Beauchet O, Hausdorff JM (2012) Gait and cognition: a complementary approach to understanding brain function and the risk of falling. $J \mathrm{Am}$ Geriatr Soc 60, 2127-2136.

[33] Liu H (Howe), Eaves J, Wang W, Womack J, Bullock P (2011) Assessment of canes used by older adults in senior living communities. Arch Gerontol Geriatr 52, 299-303.

[34] Nadkarni NK, Lopez OL, Perera S, Studenski SA, Snitz BE, Erickson KI, Mathis CA, Nebes RD, Redfern M, Klunk WE (2016) Cerebral amyloid deposition and dual-tasking in cognitively normal, mobility unimpaired older adults. $J$ Gerontol A Biol Sci Med Sci 72, 431-437. 